\title{
What will it take to prevent another Pediatric Sedation or Anesthetic Fatality?
} John E Nathan*

Adjunct Professor, Department of Pediatric Dentistry, University of Alabama, and Case Western Reserve; Clinical Associate Professor, Dept of Otolaryngology, Northwestern University Feinberg School of Medicine, USA

*Corresponding author: John E Nathan, Department of Pediatric Dentistry, University of Alabama, Birmingham and Case Western University, Cleveland, USA, Tel: 630574-7336; E-mail: jnathandds@gmail.com

Received date: June 25, 2017; Accepted date: June 28, 2017; Published date: July 05, 2017

Copyright: ( 2017 Nathan JE. This is an open-access article distributed under the terms of the Creative Commons Attribution License, which permits unrestricted use, distribution, and reproduction in any medium, provided the original author and source are credited.

\section{Editorial}

Sadly we recently learned of the tragic death of a three year old who underwent dental treatment at an out-patient surgical center in Stockton, California. This is regrettably not an uncommon occurrence over the past two decades [1]. Numerous fatalities continue to occur with the use of in-office sedation by both pediatric dental specialists and non-specialists in efforts to avoid prohibitively costly general anesthetics in hospitals or surgical centers. In this recent case, it is unclear if sedation, or general anesthesia was deployed at this surgical center for this child.

Despite these rare but recurring tragic incidents, the American Academy of Pediatric Dentistry reports that hundreds if not thousands of safe and appropriately treated, well-monitored child sedations occur on a daily basis. Documented efforts exploring cause of mishap and fatalities reveal numerous common denominators [2]. Among the causes include failure of practitioners to be sufficiently familiar with the agents being used, proper dosing, and their physiologic effects. Failure to appropriately evaluate and assess the physical status of the patient pre-operatively, intra- and post-operatively serve as common factors leading to an adverse reaction and outcome. Among the most frequent problem reported is a failure to monitor (and record) the patient as per the needs of the particular patient with respect to vital signs, protective reflexes, and the level of sedation induced. Practitioner/staff proficiency to recognize and manage an adverse reaction as well as inadequately equipped facilities to provide and enable appropriate emergency management appear as relevant factors. Exceeding toxic doses of local anesthetic in combination with sedative agents and combinations have been cited in cases of catastrophic outcome. Lastly, premature discharge of patients to non-professional custodial care and transport have contributed to post-treatment mishaps. Discharge criteria are well defined and established for patient and parent safety $[3,4]$.

Release of details will not readily become available for quite some time as this case above is under investigation by the State of California's Dept of Regulation. In fairness, there is no information as to cause whether it included an idiosyncratic and adverse reaction to conventional anesthetic delivery, undiagnosed cardiovascular anomaly, or negligence. Allegedly, a pediatric dental specialist was not involved. Nothing yet is known as to the pediatric anesthesiology expertise of the facility's anesthetist in attendance or staff of the facility.
To date, no national data bank mandates records be maintained for cases involving morbidity or mortality in dentistry, pediatric sedation or anesthesiology. Release of such data, identifying etiology, practitioners/facilities involved, and victims is rare; following cursory reporting generally these findings become buried as result of ensuing litigation and settlement. Guidelines to insure safety for children, parents, and practitioners have existed since 1985 across multiple disciplines (American Academy of Pediatric Dentistry, American Academy of Pediatrics, American Society of Anesthesiology, and American Association of Oral and Maxillofacial Surgery). Despite the existence of numerous safeguards and disciplinary guidelines and updated revisions, state licensure does not include verification of compliance standards and safety net guidelines until a tragedy occurs.

Numerous limited continuing education courses have recently emerged which offer weekend instruction on how to safely sedate child and adult patients. Attraction on the basis of profitability is a core element for these endeavors. Whether these courses offer sufficient coverage to enable safe and efficacious care given limited time commitments and hands-on experience, remains unclear. It seems plausible that experienced clinicians with existing expertise in advanced airway management and medical emergency management will find these upgraded forms of sedation helpful to refine their skills, but skepticism prevails as to the viability and safety of such for the novice seeking to add sedation to their practices. Currently no regulation appears on the horizon for this category of continuing education.

\section{References}

1. Nathan JE (2017) A Predictable and Practical Approach to avoid mishaps, adverse reactions, and Catastrophic outcomes. J Pharmacology Clin Research 2: 1-3.

2. Nathan JE (2017) Helping Anxious and Uncooperative Children to Cope with Invasive Dental Care Using Sedation: A Double Edged Sword. Oral Health and Dentistry 1: 104-106.

3. Nathan JE (2016) Morbidity and Mortality involving Pediatric sedation: Non-Compliance following Sedation Guidelines. J Surgery.

4. Nathan JE (2016) Historical and Contemporary Use of Chloral Hydrate as a sedative-hypnotic and alternatives for management of moderate to severe childhood dental anxiety and uncooperative behavior. J Pharmacology and Clinical research 1: 1-7. 